\title{
Effect of a single dose of pregabalin on herpes zoster pain
}

Christina Jensen-Dahm ${ }^{1}$, Michael C Rowbotham², Haatem Reda ${ }^{3}$, Karin Lottrup Petersen ${ }^{4 *}$

\begin{abstract}
Background: The effect of pregabalin on acute herpes zoster pain has not been previously evaluated.

Methods: In a randomized, double-blind, placebo-controlled, two-session crossover study the effect of a single oral dose of pregabalin $(150 \mathrm{mg}$ ) on pain and allodynia was evaluated in 8 subjects with herpes zoster.

Results: Over 6 hours of observation, pain decreased by a mean of 33\% with pregabalin and 14\% with placebo ( $p$ $<0.10$ ). Effects on allodynia and SF-MPQ were not significant.

Conclusions: Compared to an earlier study of gabapentin $900 \mathrm{mg}$ for acute zoster pain and allodynia that followed a nearly identical protocol, pregabalin had a similar effect on pain and was well tolerated, with no difference from placebo on sleepiness. Common side effects of light-headedness, unsteady gait, and slowed thinking were almost identical to that observed in the earlier study of gabapentin. Subject recruitment proved difficult in part due to the widespread off-label use of gabapentin and pregabalin for acute zoster pain in our region of the USA.
\end{abstract}

Trial Registration: ClinicalTrials.gov Identifier: NCT00352651

\section{Introduction}

Herpes zoster (HZ), the reactivation of latent varicella zoster virus in sensory ganglia, produces a painful, unilateral, dermatomal rash. We have previously demonstrated that a single oral dose of gabapentin $(900 \mathrm{mg}$ ) reduces pain and allodynia during HZ [1]. Both pregabalin and gabapentin bind to the $\alpha 2 \delta$-subunit of the calcium channel [2], but no head-to-head efficacy comparisons have been made. In this randomized, placebocontrolled, two-session, crossover study we evaluated the effect of a single dose of $150 \mathrm{mg}$ of pregablin on pain and allodynia in subjects with $\mathrm{HZ}$ using a nearly identical protocol.

\section{Materials and methods Subjects}

All subjects provided informed written consent. The study was approved by the UCSF Committee on Human Research. Subjects were medically stable adults with onset of unilateral HZ during the previous 6 weeks and

\footnotetext{
* Correspondence: Karin.Petersen@ucsf.edu

${ }^{4}$ University of California San Francisco, Department of Neurology, CA, USA

Full list of author information is available at the end of the article
}

continuing pain $\geq 40$ on a $100 \mathrm{~mm}$ pain visual analogue scale (VAS; $0=$ no pain, $100=$ worst imaginable pain) at the screening visit. Exclusion criteria included: prior pregabalin use, nerve block therapy within the last 48 hours, use of gabapentin within the last 72 hours, unrelated severe pain and clinically significant medical or psychiatric disease. Stable doses of oral NSAIDs, acetaminophen, opioids, anticonvulsants (except pregabalin or gabapentin) or antidepressant were allowed and could be taken up to 2 hours prior to medication visits. Subjects were not allowed to use topical analgesics 12 hours prior to medication visits.

\section{Visits and measures}

Following the screening visit, the two medication visits (V1 and V2) were separated by at least 48 hours. Medication was administered double-blind as a single dose of $150 \mathrm{mg}$ of pregabalin or identical-appearing placebo. Randomization was computer generated and managed by a study pharmacist not otherwise involved in the study. Subjects rated their pain on the $100 \mathrm{~mm}$ pain VAS. Allodynia area was mapped by stimulating the skin with a 1 -inch foam brush going from normal to affected skin. Within the area of greatest pain, the intensity of allodynia 
on a $100 \mathrm{~mm}$ allodynia VAS scale $(0=$ not unpleasant, 100 = most unpleasant imaginable) was rated after 3 strokes with the foam brush. Subjects rated side-effects (sleepiness, light-headedness or dizziness, unsteady gait, nausea and vomiting) on a $0-3$ scale $(0=$ none, $1=$ mild, 2 = moderate, 3 = severe). Pain, allodynia intensity and side-effects were rated prior to medication and every 30 minutes for 6 hours. Allodynia areas were mapped at 1,2,3, and 6 hours post-medication. The Short Form McGill Pain Questionnaire was administered pre-medication and 2 hours post-medication [3].

\section{Statistical analysis}

In a previous study a single $900 \mathrm{mg}$ dose of oral gabapentin reduced $\mathrm{HZ}$ pain by approximately $33 \%$ (compared to placebo). The standard deviation of the change was $50 \%$, resulting in a standardized effect size $(E / S)$ of 0.7 . With a two-tailed $\alpha$ of 0.05 , and a $\beta$ of 0.2 , the sample size desired is 34 . The primary efficacy variable, VAS pain, was analyzed using a model that included fixed effects for treatment, age, treatment order, sex, and baseline pain and a random subject effect to account for correlated data between the two treatments. A similar analysis was used for other, secondary, repeated measures. Post hoc analyses of percent reduction from baseline at each time point for the primary efficacy variable used the Wilcoxon signed-rank test. SF-MPQ (total score) results were analyzed with Wilcoxon signed-rank test.

\section{Results}

Between January 2006-March 2008, 334 potential subjects were telephone screened, with 26 attending a screening visit (Figure 1). Eight subjects were dosed and all completed the study. The study was closed in March 2008 due to the slow pace of enrollment. Baseline characteristics are shown in Table 1 and changes in pain ratings during the two drug administration sessions are shown in Figure 2. Overall, there was a trend toward greater reduction in pain after pregabalin administration than after placebo (placebo - pregabalin: -19.0\% [95\% CI: $-42.5 \%$ to $+4.5 \%] ; p=0.096$. Analysis of the individual time points for the difference between pregabalin and placebo revealed significance at 1.5 hours after medication administration $(-23.5 \%$ [ $-44.5 \%$ to $-2.5 \%]$; $p$ $=0.034)$, and trends at 2.5 hours $(-31.9 \%$ [-70.1\% to $+6.3 \%] ; \mathrm{p}=0.099)$ and 5 hours $(-43.7 \%[-91.2 \%$ to $+3.9 \%] ; \mathrm{p}=0.076)$. Relative to placebo, we found a trend towards greater maximal pain reduction with pregabalin $(-22.9 \%[-52.0$ to +6.0$) ; \mathrm{p}=0.100)$. Changes in severity and area of allodynia, and the change in SFMPQ scores, were not significantly different between the pregabalin and placebo sessions (data in Table 2). Side-effects were well tolerated (Table 2).

\section{Discussion}

Pregabalin has not been previously studied for acute zoster pain. Pregabalin has been studied in Post Herpetic Neuralgia (PHN), the chronic neuropathic pain condition afflicting some patients after an episode of herpes zoster (HZ). In multiple clinical trials for PHN, pregabalin has been proven effective at daily doses of $150 \mathrm{mg} /$ day and higher [4,5]. Efficacy is demonstrable within a few days of starting treatment [4]. In one large trial, changes in allodynia severity correlated with changes in ongoing pain

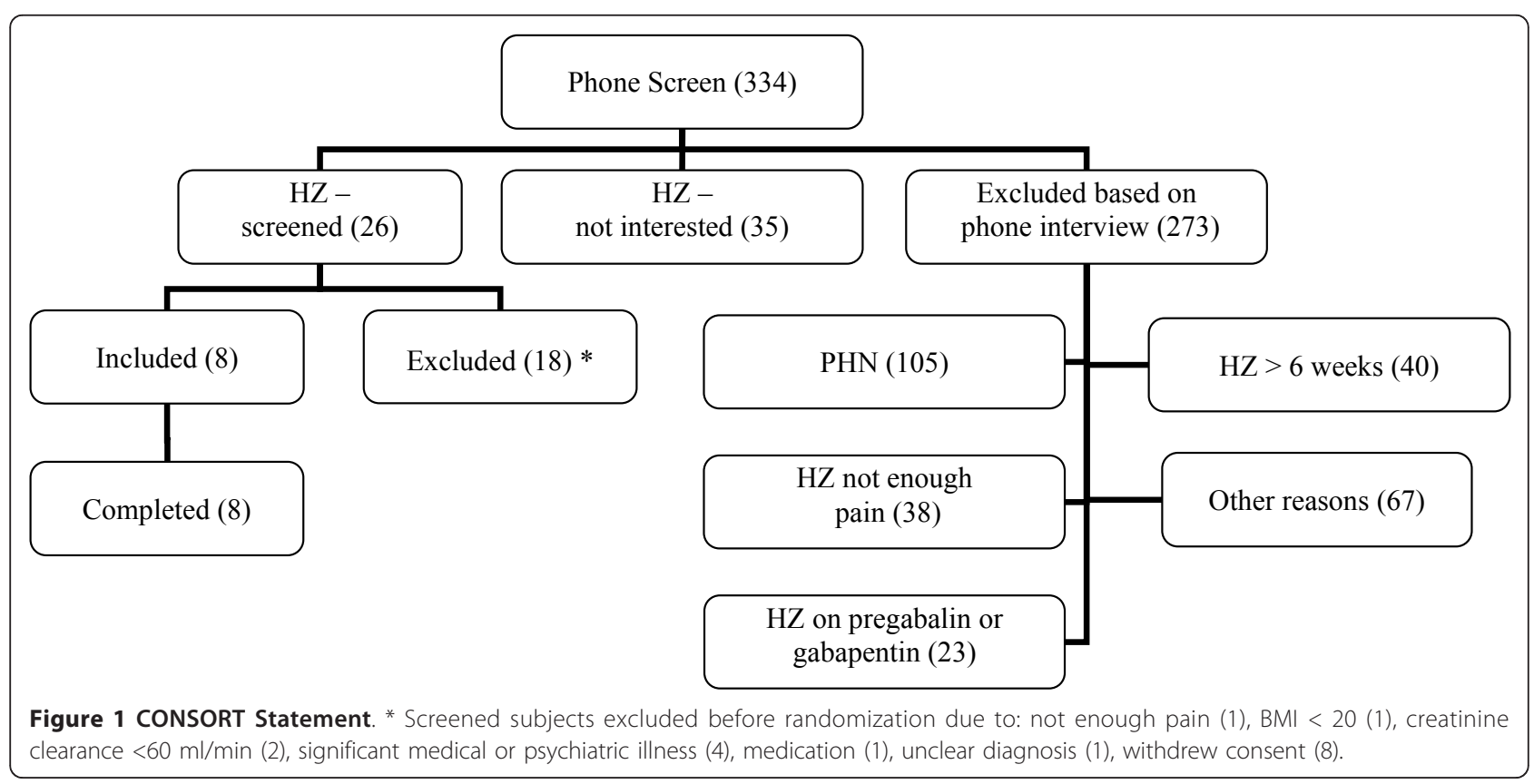


Table 1 Baseline demographics, pain and allodynia

\begin{tabular}{lcc}
\hline & Placebo first group, $\mathbf{n}=\mathbf{3}$ & Pregabalin first group, $\mathbf{n}=\mathbf{5}$ \\
\hline Demographics & $77(73-84)$ & $65(22-76)$ \\
\hline Age; median (range) & $33 \%$ & $40 \%$ \\
\hline \% Female & 0 & 1 \\
\hline Distribution of Zoster & 1 & 1 \\
\hline Trigeminal & 2 & 3 \\
\hline Cervical & & 0 \\
\hline Thoracic & $100 \%{ }^{\mathrm{a}}$ & \\
\hline Severity of rash & & $80 \%$ \\
\hline Mild & $31(31-37)$ & $20 \%$ \\
\hline Moderate & $2(2-5)$ & $\left(10-45^{b}\right)$ \\
\hline Severe & $63(20-90)$ & $2(2-5)$ \\
\hline Days between outbreak and study entry; median (range) & $48(16-90)$ \\
\hline Days between visit 1 and visit 2; median (range) & $35(17-79)$ & 30 (9-94) \\
\hline Pain at V1, 0-100; median (range) & $51(19-74)$ & $37(10-84)$ \\
\hline Pain at V2, 0-100; median (range) & $20(15-48)$ & $34(0-70)$
\end{tabular}

${ }^{\mathrm{a} O n e ~ s u b j e c t ~ m i s s i n g, ~}{ }^{\mathrm{b}}$ One subject included at 45 days; met all other inclusion criteria.

[5]. Despite the very small number of subjects in the present study, there was a trend towards a greater reduction in pain after a single dose of $150 \mathrm{mg}$ of pregabalin. Using the data given in table 2 , the standardized effect size is 1.16. With this effect size and a two-sided $\alpha$ of 0.05 and $\beta$ of 0.2 , a future study would need only 18 subjects to reach significance. A significant effect of pregabalin on allodynia severity or area was not demonstrated.

A previous study of nearly identical design conducted in our unit included 26 subjects with $\mathrm{HZ}$ and showed

\section{Pain Visual Scale over 6 hours}

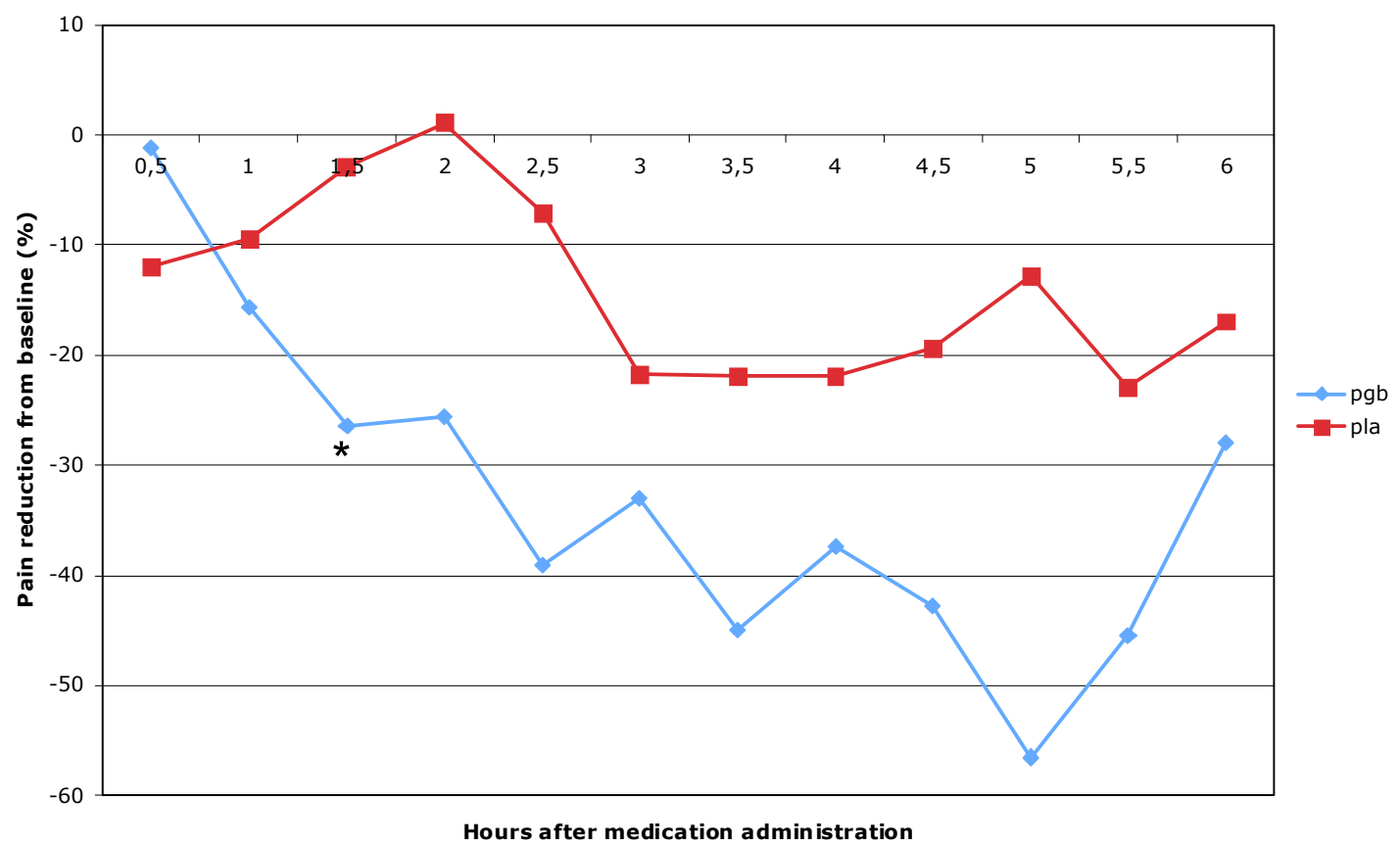

Figure 2 Percent reduction in median pain VAS (placebo and pregabalin) at specific time points after medication administration. A larger negative number means greater reduction in pain. ${ }^{*} p<0.05$. 
Table 2 Outcome Measures

\begin{tabular}{lll}
\hline Outcome (Mean and $\mathbf{9 5} \% \mathbf{C l}$ ) & Pregabalin, $\mathbf{n}=\mathbf{8}$ & Placebo, $\mathbf{n}=\mathbf{8}$ \\
\hline Overall Pain percent reduction & $33.0(13.2-52.9)$ & $14.0(-5.8$ to 33.8$)$ \\
\hline Overall Pain VAS reduction & $14.7(5.1-24.3)$ & $8.5(-1.1$ to 18.1$)$ \\
\hline Allodynia severity, overall percent reduction & $25.2(0.9-49.5)$ & $30.7(7.5-53.9)$ \\
\hline Allodynia area, overall percentage reduction & $-1.4(-59.6$ to 56.8$)$ & $-12.4(-70.8$ to 45.9) \\
\hline Total SF McGill (0-45), premedication & $12.4(8-25)$ & $12.3(7-26)$ \\
\hline Total SF McGill (0-45), post medication & $7.25(3-22)$ & $8.9(6-16)$ \\
\hline Side-effects; 0 to 3 & $0.7(0-1.2)$ & $0.7(0-1.7)$ \\
\hline Sleepiness & $0.5(0-1.6)$ & $0.2(0-1.1)$ \\
\hline$\quad$ Light-headedness & $0.2(0-1.2)$ & $0.05(0-0.4)$ \\
\hline Unsteady gait & $0.2(0-1)$ & 0 \\
\hline Slowed thinking & $0.01(0-0.08)$ & $0.1(0-0.5)$ \\
\hline Headache & $0.1(0-1)$ & $0.09(0-0.4)$ \\
\hline Nausea & 0 & 0 \\
\hline$\quad$ Vomiting & $0.01(0-0.08)$ & 0 \\
\hline Blurry vision & $1.7(0.2-3.9)$ & $1.1(0-4.1)$ \\
\hline Total Side-effect score & &
\end{tabular}

that a single dose of $900 \mathrm{mg}$ gabapentin reduced both pain and allodynia [1]. In the present study, the maximum percent reduction in pain with pregabalin was $63 \%$ compared to $40 \%$ with placebo, quite similar to the $66 \%$ reduction with gabapentin and $33 \%$ reduction with placebo in the earlier study. The pattern of side effects was quite similar between the two studies, with the noteworthy exception that sleepiness was greater than placebo with gabapentin, and identical to placebo with pregabalin. Trials using a single relatively large dose have only limited ability to predict longer term treatment results. The recent multicenter trial by Dworkin and colleagues in 87 subjects treated within 6 days of zoster rash onset compared placebo, oxycodone, and gabapentin up to $600 \mathrm{mg}$ three times a day [6]. Gabapentin therapy showed a trend for efficacy in the first week which was completely lost as the trial continued, while pain reduction with oxycodone remained significantly greater than with placebo. However, the natural history of the decline in pain complicates longer-term trials in $\mathrm{HZ}$ patients. At treatment day 9 in the Dworkin et al study, even 'worst pain' in the placebo group was already below 4/10.

When evaluating efficacy of new compounds for neuropathic pain in $\mathrm{HZ}$ and $\mathrm{PHN}$, baseline pain ratings should be at least moderate to severe to allow measurement of pain relief. Herpes zoster is much more common than PHN, with well over a million new cases each year in the USA, and in the study of Helgason and colleagues, between $7 \%-30 \%$ of subjects reported their acute zoster pain as moderate to severe [7]. The incidence of moderate to severe PHN after HZ is lower; in the landmark vaccine study by Oxman and colleagues, the incidence of PHN (worst pain $>/=3 / 10$ ) was $5.1 \%$ at
6 months in placebo vaccinated subjects aged 60 or older [8]. However, as PHN pain is chronic and often insufficiently treated, the point prevalence of patients looking to participate in clinical trials may indeed be larger than the point prevalence of patients with moderate-severe $\mathrm{HZ}$ pain, many of whom are hoping the pain will spontaneously subside. When considering whether to study HZ or PHN, it should also be kept in mind that the neurodestructive and inflammatory aspects of herpes zoster might make this condition respond differently to treatment than chronic neuropathic pain disorders such as PHN and painful diabetic neuropathy.

Recruitment is always an important issue in clinical trials, and this study demonstrates that recruitment challenges can be severe. During an enrolment period of more than 2 years, we phone screened 334 subjects yet dosed a total of 8 . Only 162 were within the specified time frame after rash onset for study inclusion and others had insufficient pain. Unlike trials of investigational compounds not otherwise available, many otherwise eligible subjects declined to participate because pregabalin could be obtained from their primary physician, or they had current or prior treatment with pregabalin or gabapentin for their HZ pain (frequently initiated at the time of zoster diagnosis). We used only print newspaper and online advertising (Craigslist). Recruiting within emergency rooms, primary care clinics, and dermatology practices might yield untreated and potentially eligible subjects within the first few days of rash onset.

\section{Conclusions}

In summary, this small placebo-controlled crossover trial of a single relatively large dose of pregabalin 
showed a trend for reducing acute zoster pain. The effect size was similar to that observed in a prior study of gabapentin in a much larger group of acute zoster patients. Greatly complicating recruitment for this placebo-controlled study was the widespread clinical practice of 'off-label' initiation of pregabalin or gabapentin therapy at the time of zoster diagnosis.

\section{Acknowledgements}

This study was funded by an investigator-initiated grant from Pfizer, Inc. The study was designed and conducted independently by the authors.

Collection, management, analyses, and interpretation of the data were done in collaboration with Dr. Alan Bostrom, Department of Epidemiology and Biostatistics, UCSF. All data are owned and controlled by the authors. The manuscript was prepared by the authors and was not subject to review or approval by Pfizer.

\section{Author details}

'Department of Neurology University of Copenhagen, Rigshospitalet, Denmark. ${ }^{2}$ California Pacific Medical Center Research Institute, CA, USA.

${ }^{3}$ Department of Neurology, Mayo Clinic, Rochester, Minnesota, USA.

${ }^{4}$ University of California San Francisco, Department of Neurology, CA, USA.

\section{Authors' contributions}

CJD conducted the study and drafted the manuscript. KLP designed the study, performed the statistical analysis and helped draft the manuscript. MCR was study physician, participated in study design, and helped draft the manuscript. HR helped design and conduct the study. All authors read and approved the final manuscript.

\section{Competing interests}

The authors declare that they have no competing interests.

Received: 14 June 2010 Accepted: 28 February 2011

Published: 28 February 2011

\section{References}

1. Berry JD, Petersen $\mathrm{KL}: \mathrm{A}$ single dose of gabapentin reduces acute pain and allodynia in patients with herpes zoster. Neurology 2005, 65(3):444-7.

2. Taylor CP: Mechanisms of analgesia by gabapentin and pregabalincalcium channel alpha2-delta [Cavalpha2-delta] ligands. Pain 2009, 142(1-2):13-6.

3. Strand LI, Ljunggren AE, Bogen B, Ask T, Johnsen TB: The Short-Form McGill Pain Questionnaire as an outcome measure: test-retest reliability and responsiveness to change. Eur J Pain 2008, 12(7):917-25.

4. Dworkin RH, Corbin AE, Young JP Jr, Sharma U, LaMoreaux L, Bockbrader H, Garofalo EA, Poole RM: Pregabalin for the treatment of postherpetic neuralgia: a randomized, placebo-controlled trial. Neurology 2003, 60(8):1274-83.

5. Stacey BR, Barrett JA, Whalen E, Phillips KF, Rowbotham MC: Pregabalin for postherpetic neuralgia: placebo-controlled trial of fixed and flexible dosing regimens on allodynia and time to onset of pain relief. J Pain 2008, 9(11):1006-17.

6. Dworkin RH, Barbano RL, Tyring SK, Betts RF, McDermott MP, PennellaVaughan J, Bennett GJ, Berber E, Gnann JW, Irvine C, Kamp C, Kieburtz K, Max MB, Schmader KE: A randomized, placebo-controlled trial of oxycodone and of gabapentin for acute pain in herpes zoster. Pain 2009, 142(3):209-17.

7. Helgason SI, Petursson G, Gudmundsson S, Sigurdsson JA: Prevalence of postherpetic neuralgia after a first episode of herpes zoster: prospective study with long term follow up. BMJ 2000, 321:794-6.

8. Oxman MN, Levin MJ, Johnson GR, Schmader KE, Straus SE, Gelb LD, Arbeit RD, Simberkoff MS, Gershon AA, Davis LE, Weinberg A, Boardman KD, Williams HM, Zhang JH, Peduzzi PN, Beisel CE, Morrison VA, Guatelli JC, Brooks PA, Kauffman CA, Pachucki $C T$, et al: A vaccine to prevent herpes zoster and postherpetic neuralgia in older adults. N Engl J Med 2005, 352(22):2271-84 doi:10.1186/1745-6215-12-55

Cite this article as: Jensen-Dahm et al:: Effect of a single dose of pregabalin on herpes zoster pain. Trials 2011 12:55.

\section{Submit your next manuscript to BioMed Central and take full advantage of:}

- Convenient online submission

- Thorough peer review

- No space constraints or color figure charges

- Immediate publication on acceptance

- Inclusion in PubMed, CAS, Scopus and Google Scholar

- Research which is freely available for redistribution 